\title{
Autopercepção do sofrimento psíquico em indivíduos com sintomatologia depressiva e comportamento suicida
}

Giovanna Vallim Jorgetto ${ }^{1}$ (D), João Fernando Marcolan ${ }^{1}$

\begin{abstract}
RESUMO
Objetivo do estudo: Analisar a autopercepção do sofrimento psíquico em indivíduos com sintomatologia depressiva e sua relação com comportamento suicida em população geral adulta. Metodologia: Pesquisa exploratória, descritiva, qualitativa, com o uso da Análise de Conteúdo temático. Realizadas entrevistas por questionário semiestruturado com 200 participantes acima de 18 anos, domiciliados em Poços de Caldas/MG, entre janeiro de 2017 a outubro de 2018. Resultados: Participantes apresentaram não percepção do quadro depressivo e desejo de morte como saída frente ao sofrimento mental ocasionado pela depressão; justificaram para o desenvolvimento da depressão as perdas afetivas, medo, desespero, ausência de apoio familiar, ruminação de ideias, relações familiares conflituosas, perda da autoestima e solidão. Conclusões: Autopercepção da sintomatologia depressiva esteve relacionada a não ter percepção de depressão ou admissão do transtorno e tristeza pelas lembranças despertadas. Relação entre sintomatologia depressiva e comportamento suicida se fez presente.
\end{abstract}

Palavras-chave: Depressão, Comportamento suicida, Saúde mental, Percepção, Conhecimento. 


\section{INTRODUÇÃO}

Segundo Nóbrega, Leal, Marques, Vieira ${ }^{1}$, embora a depressão não seja uma doença silenciosa, é pouco diagnosticada na prática clínica diária, e estima-se que apenas $34 \%$ das pessoas com depressão procurem ajuda especializada e apenas um terço encontrem a ajuda necessária. Na maioria das vezes, as pessoas são incapazes de reconhecer ou nomear o seu transtorno e, frequentemente, apresentam queixas somáticas, tais como, cefaleia, inapetência e problemas de estômago, diminuição da libido e ereção, além de falta de energia, traduzidas rapidamente em possibilidades de diagnóstico de alguma doença orgânica específica².

Soma-se a falta de habilidade e capacitação dos profissionais de saúde em conhecer o indivíduo no que concerne a sua história de vida, contexto pessoal, familiar, profissional, social, pensamentos, sentimentos e comportamentos, a fim de delimitar a hipótese de sofrimento psíquico e o manejo adequado deste na rede de atenção psicossocial ${ }^{3,4}$.

A depressão, quando não diagnosticada ou tratada adequadamente, torna-se um dos principais fatores de risco para o comportamento suicida que engloba os pensamentos de autodestruição, passando por ameaças, gestos, tentativas e, finalmente, o próprio suicídio em $\mathrm{si}^{5}$. Ressalta-se que a relação entre depressão e comportamento suicida está em processo de retroalimentação pela sintomatologia depressiva como humor deprimido, isolamento social, anedonia, alterações humor, apetite, sono, agitação ou lentidão psicomotora, fadiga e perda de energia, sentimento de inutilidade e ideação de culpa excessiva ou inadequada, capacidade diminuída de pensar ou concentrar-se ou indecisão quase todos os dias a desencadear os pensamentos de morte recorrentes, gerando, assim, círculo vicioso que aumenta a sintomatologia depressiva e a probabilidade de suicídio. Desta forma, este participante afasta-se dos amigos e pessoas mais próximas, apresentando ainda desinteresse pelo trabalho, lazer e qualquer outra atividade do dia a dia².

A presença de ideação suicida é, por si só, um importante sinal de sofrimento psíquico e exige atenção redobrada na avaliação clínica. Um transtorno psiquiátrico pode estar presente, a necessitar de pronto reconhecimento e tratamento adequado ${ }^{2,6}$.

Desta forma, o presente estudo teve como objetivo analisar a autopercepção acerca do sofrimento psíquico em indivíduos com sintomatologia depressiva e comportamento suicida em população geral da cidade de Poços de Caldas/MG.

\section{MÉTODO}

\section{Delineamento e participantes}

Trata-se de estudo descritivo exploratório, com abordagem qualitativa, realizado na cidade de Poços de Caldas/MG, município de médio porte do sul do estado. O período da coleta de dados se deu entre janeiro de 2017 a outubro de 2018.

A população foi composta de residentes e domiciliados em Poços de Caldas/MG. A amostra probabilística foi aleatoriamente estratificada e definida por conveniência a 200 indivíduos, escolhida aleatoriamente entre o centro e os três pontos cardeais da cidade (Sul, Leste e Oeste), mantida a proporção de 50 indivíduos por área geográfica. A exclusão da Zona Norte se deu ao fato de esta ser área de mata preservada no Município e não possuir habitações. A quantidade de entrevistas foi dividida pela quantidade de bairros das regiões. Houve cuidado para que houvesse divisão igualitária entre a quantidade de entrevistados por bairro. Houve sorteio da rua de cada bairro e a coleta teve início pelo número de residência mais baixo da rua. Após, a abordagem foi realizada do outro lado da rua, respeitada a alternância de duas casas entre um entrevistado e outro. Tal cuidado se fez necessário a fim de evitar comunicação entre os vizinhos e contaminação das respostas dos participantes. Os critérios de inclusão foram: indivíduos de ambos os sexos, com idade acima de 18 anos, residentes e domiciliados em Poços de Caldas/MG, em tempo igual ou superior há dose meses e que apresentassem condições cognitivas para participar do estudo.

\section{Procedimento coleta de dados}

Quanto ao procedimento de coleta de dados, os instrumentos utilizados foram escalas psicométricas para sintomatologia depressiva, aplicadas na seguinte ordem: Inventário de Depressão de Beck, Escala de Avaliação para Depressão de Hamilton e Escala de Avaliação para Depressão de Montgomery - Asberg. Para essas escalas, foi solicitado ao participante que respondesse para cada item qual alternativa descrevia melhor seus sentimentos na última semana, incluído o dia da pesquisa. Passou-se a seguir a entrevista com aplicação de questionário semiestruturado com itens referentes ao perfil sociodemográfico dos participantes, dados a respeito de antecedentes de depressão e por questões formuladas tendo como pressuposto os objetivos da pesquisa: Qual é 
a sua percepção sobre o sofrimento psíquico que relatou/ foi detectado pelas escalas e Descreva a sua percepção sobre a sintomatologia depressiva na relação com o comportamento suicida. As entrevistas acontecerem no domicílio do participante, de maneira reservada, o que garantiu o sigilo das informações, após explicação sobre o que compreendia a pesquisa e, sendo resolvidas as dúvidas, após terem concordado em participar, foi solicitado que firmasse compromisso pela assinatura do Termo de Consentimento Livre e Esclarecido. A entrevista foi gravada em áudio para a transcrição na íntegra.

\section{Análise dos dados}

Os dados coletados por entrevistas foram analisados pela análise de conteúdo temático, contempladas as fases de pré-análise, exploração do material e tratamento dos resultados, inferência e interpretação?.

\section{Aspectos éticos}

Em relação aos aspectos éticos, garantiu-se respeito às normas que regulamentam a pesquisa com seres humanos. A pesquisa se deu após aprovação do Comitê de Ética em Pesquisa da Universidade Federal de São Paulo, sob parecer n. ${ }^{\circ}$ 1.813.770/2016.

\section{RESULTADOS}

\section{Caracterização sociodemográfica dos participantes}

Foram 200 participantes deste estudo e a análise do perfil sociodemográfico evidenciou maioria composta pelo gênero feminino, idade entre 29 a 39 anos e acima de 60 anos, branca, católica, casada, com filhos (maioria referiu dois filhos), ensino fundamental incompleto e ensino médio completo, com até três pessoas vivendo no domicílio, vivendo com companheiros/filhos/ cônjuges, exercendo atividade laboral não remunerada, desempregada e aposentada, com renda individual e familiar de 2 a 5 salários mínimos e referindo como recreação estar com os amigos.

Avaliação das escalas psicométricas utilizadas para avaliação dos sintomas depressivos.

Quanto às três escalas psicométricas aplicadas para avaliação dos sintomas depressivos, a maioria dos participantes com sintomatologia prévia referida apresentaram escores de depressão leve, enquanto o grupo com ausência de sintomas prévios apresentou percentuais maiores para intensidade moderada e grave. Houve ainda um terço dos participantes acima de 61 anos com escores positivos para sintomatologia depressiva, principalmente em intensidade leve e moderada, através das escalas psicométricas utilizadas neste estudo.

O comportamento suicida esteve presente em todos os participantes que tiveram sintomatologia depressiva apontada pelas escalas, e de forma mais acentuada para aqueles que apresentaram escores maiores nos grupos moderado e grave.

Após a transcrição das questões norteadoras, os discursos dos participantes foram analisados e determinadas as unidades de registro que continham significado que abarcassem os objetivos propostos para este estudo e demais pontos de interesse. Desta forma, emergiram duas categorias temáticas, sendo uma relacionada à percepção ou ausência desta pelos participantes acerca dos sintomas depressivos e a segunda sobre a relação entre sintomatologia depressiva e comportamento suicida.

Percepção ou ausência desta pelos participantes acerca dos sintomas depressivos.

Emergiram nesta categoria questões relacionadas à percepção ou ausência desta pelos participantes sobre o sofrimento psíquico. A ausência se deu relacionada à percepção do sofrimento psíquico e sensação de piora dos sintomas depressivos ocorrer somente após entrevistas e aplicação de escalas psicométricas, quando reviveram momentos de perdas afetivas, separações conjugais por traição e desemprego, entre outras. Alguns dos participantes verbalizaram alívio em poder externar suas histórias pessoais ao entrevistador. Desta forma, aqui foram elencados quatro itens temáticos, sendo: Percepção de estar com depressão, Percepção de sensação de alívio após entrevistas, Percepção de piora da sensação de depressão ao ser entrevistado (a) e Percepção de não estar com depressão.

\section{Percepção de estar com depressão}

Quanto à percepção de estar com depressão, ao analisarmos o conteúdo das falas dos participantes, emergiu para alguns, a consciência de sofrer de depressão. As falas abaixo demostram consciência dos mesmos frente aos sintomas depressivos, atrelados a problemas pessoais ou de vida, que desencadearam sentimentos de solidão, tristeza e isolamento social, entre outros.

$E 12=$ No momento sinto uma enorme solidão no meu coração e esta solidão está me matando, acabando com minha vontade de viver.

$E 24=$ Senti que estou deprimido. Não consigo trabalhar no que gosto, não gosto do meu emprego... 
$E 49=$ No momento estou tão depressiva que não quero mais me relacionar com a minha família e amigos. Não consigo ficar perto da minha família, mas estou em tratamento no CAPS.

$E 67=$ Gostei de responder às perguntas, pois muitas vezes me sinto muito sozinha e faz bem para mim conversar com as pessoas. Acho que depois de responder estas perguntas estou um pouco deprimida.

Percepção de sensação de alívio após entrevistas. Para a percepção de sensação de alívio após entrevistas, descrita pelos participantes após responderem às questões norteadoras e instrumentos de estudo, esta foi verbalizada pela experiência positiva de compartilhamento de suas histórias pessoais com o entrevistador.

$E 16=$ Posso dizer com segurança que me senti bem ao responder as perguntas. Deu uma aliviada nos meus sentimentos. Foi ótimo falar com você.

$E 17=$ Fiquei feliz ao expressar o que sinto, me fez bem.

E21 $=$...ao compartilhar minhas histórias, me senti melhor...

$E 22$ = Senti um alerta e alívio ao responder as perguntas. Precisa fazer estas perguntas para mais pessoas...

$E 23=$ Me senti bem ao responder as perguntas...

E25 $=$ Me senti bem em responder. Através dessas perguntas, pude, de alguma forma, expressar o que realmente sinto, pois é uma batalha árdua e difícil a depressão. Só conseguimos superar através de ajuda médica e familiar.

Percepção de piora da sensação de depressão ao ser entrevistado(a).

Houve ainda a percepção de piora da sensação de depressão ao ser entrevistado(a), externado por verbalização de recordações com sofrimento psíquico ao longo da vida, a exemplo de separações conjugais, perda afetivas, entre outros.

$E 1=\ldots$ me senti triste, pois lembrei das coisas ruins que aconteceram. Minha separação. A traição do meu marido...

$E 3=$ Fiquei triste em pensar em coisas ruins que aconteceram neste período triste da minha vida. Minha vontade de morrer.

E11 = Fiquei triste, pois me lembrei dos piores momentos da minha vida, morte do meu irmão e de quando fiquei depressiva e não fazia outra coisa a não ser chorar.

$E 45=$ Fiquei abatido porque foi uma fase muito difícil que relembrei quando estava desempregado. Mas não acho que estou deprimido. Agora estou bem, trabalhando e tudo entrando nos eixos.

Percepção de não estar com depressão.

Porém, o que mais chama a atenção, e se torna preocupante, foi a ausência de percepção da depressão, mesmo após a aplicação das escalas detectarem presença de sintomatologia depressiva em níveis leve, moderado e grave. Os participantes referiram que tais sentimentos verbalizados, como tristeza e humor deprimido, tinham relação com as circunstâncias vividas no cotidiano e não com o quadro depressivo já instalado.

E5 = Acho que são circunstâncias do momento o que estou sentindo. Não estou doente, mas tudo o que passo com meu marido alcoólatra e filho drogado me deixam assim.

$E 56=$ Não sentia que estava deprimida. Mas pensando bem, posso estar um pouquinho deprimida sim. Me sinto bem triste às vezes.

$E 58=$ Não. Acho que tem dias que a gente se sente mais para baixo e tem pensamentos e sentimentos ruins que ficam na cabeça o tempo todo e quando lembra de coisas ruins que aconteceu na vida fica com este sentimento ruim.

E61 = Acho que não estou não. Só me sinto triste às vezes e decepcionado com a vida.

\section{Percepção dos participantes sobre a relação entre sintomatologia depressiva e comportamento suicida}

Sobre a relação entre sintomas depressivos e comportamento suicida, foram relatados que a própria percepção da severidade da depressão, assim como sentimentos de desesperança, comorbidades psiquiátricas (síndrome do pânico e transtorno da ansiedade), desemprego, excesso de trabalho e ambiente laboral psiquicamente desgastante como fatores que desencadearam ou agravaram o comportamento suicida.

Evidenciou-se nos discursos o senso de desenvolvimento do quadro depressivo e sua relação com o comportamento suicida de que a morte é a solução frente ao intenso sofrimento psíquico desencadeado pelos sintomas vivenciados.

E2 = Sinto que a depressão é uma doença que não tem sentido, as pessoas julgam, mas não imaginam o quanto é difícil, ela acaba com a gente dia a dia. $A$ vontade de se matar é porque a pessoa sente que seria um alívio, o fim para a doença, que na verdade acaba mesmo sendo o fim da vida de muitos.

https://www.revistas.usp.br/rmrp 
E13= Depressão é uma doença devastadora que acaba com a pessoa que está depressiva e com as pessoas que convivem com o depressivo. Tornase tudo difícil, tudo complicado. Dá vontade de se trancar em um quarto escuro e não sair mais daquele lugar até você morrer e não dá mais sofrimento para ninguém.

$E 7=A$ depressão é uma coisa sem explicação. Não sei falar, acho que não tem solução. Quando está muito forte, como estou com pensamento só pessimista, acho que não vale a pena mais viver porque não tenho prazer em desfrutar de nada fora do meu quarto. Só quero ficar trancada e pensando que poderia ser diferente se meu filho não tivesse se matado. Sei que tenho outro filho e não deveria estar desse jeito, pois ele também depende de mim, mas não consigo, estou tentando, mas cada dia que passa fico mais triste e com vontade de morrer. Não consigo sair disso, vivo a base de medicação para dormir...

E43= Acho que a depressão é quando a pessoa perde o amor próprio e quando isso acontece a pessoa se afunda e surge a vontade de se matar.

$E 66=A$ depressão é uma fraqueza, uma falha do ser humano, e quando se encontra fraco e falha surge a vontade de tirar a própria vida como forma de alivio.

\section{DISCUSSÃO}

Quanto à caracterização sociodemográfica, ressalta-se que tais dados, em parte, vão ao encontro à literatura que coloca como fatores predisponentes a sintomatologia depressiva e comportamento suicida ser mulher, adulto jovem ou idoso, baixa escolaridade, desemprego e baixa rendaa, $2,6,8,9$. Porém, houve discrepância nos dados encontrados neste estudo no que se refere ao estado civil e cor, com os dados encontrados na literatura, que trazem como fatores de risco para depressão e suicídio solteirice e viuvez, atreladas à solidão e perda de rede de apoio, e a cor negra e parda atrelada tanto no Brasil quanto nos dados mundiais a maior vulnerabilidade social, sendo que tal vulnerabilidade torna-se fator de risco para sintomatologia depressiva e comportamento suicida ao longo da vida, inclusive por menor acesso a serviços de saúde ${ }^{2,9}$.

No que se refere à percepção do estado depressivo, estudo realizado por Peluso, Blay ${ }^{10}$ na cidade de São Paulo, com mais de 500 entrevistados, mostrou que a maior parte das respostas foi agrupada como problemas de saúde mental $(48,0 \%)$, problemas pessoais ou de vida $(37,6 \%)$ e como problemas físicos $(5,4 \%)$, dados parecidos com os obtidos em nosso estudo, embora aqui os relatos estejam fortemente relacionados aos problemas pessoais ou de vida. Neste mesmo estudo, em relação à questão sobre identificação como transtorno mental, 19,2\% da amostra acreditava que a situação descrita era de transtorno mental, dado superior ao de nosso estudo em que $12,5 \%$ dos participantes reconheceu seus sintomas depressivos.

Ressaltamos no estudo conduzido por Viana, Andrade ${ }^{11}$, que o baixo nível de escolaridade foi associado à preferência por causas de natureza espiritual/moral e biológica da depressão, dados que vão ao encontro aos nossos, visto que temos encontrado verbalização importante de fatores de risco para depressão atrelada a ausência de fé, e termos em nosso estudo, expressiva maioria dos participantes autodeclarada entre analfabetos e com ensino médio completo, com significância estatística na relação de baixa escolaridade e sintomas depressivos.

Em estudo realizado por Lin et al. ${ }^{12}$ sobre percepção e expressão de sintomas depressivos, o padrão de percepção da depressão caracterizou-se por solidão, isolamento social, afastamento da família e dificuldades no trabalho, dados estes similares aos encontrados nos relatos acima.

Ao ter oportunidade de descrever seus estados mentais, seus problemas e sentimentos a outra pessoa, o próprio participante passa a perceber mais claramente o que está sentindo e, com frequência, isso o ajuda a colocar em perspectiva seus problemas, o que traz certa sensação de controle e distanciamento entre o participante e o problema que se tornou objeto de seu discurso ${ }^{13}$. Este foi $o$ caso das falas dos participantes e nisso reside uma parte da importância da escuta terapêutica, em que o participante pode ser ouvido, sem julgo de valores, assim como ouvir a si mesmo e, ao mesmo tempo, raciocinar sobre seu problema ${ }^{14}$.

O participante, ao verbalizar o que sente, fica aliviado por poder expressar verbalmente seus sentimentos e pensamentos de maneira adequada, tende a aumentar o sentimento de satisfação, tão importante na recuperação frente aos sintomas depressivos ${ }^{15}$.

Ressaltamos que em estudo desenvolvido por Moll et al. ${ }^{16}$, os participantes com depressão e outros com transtorno bipolar confirmaram que 
o simples fato de finalmente terem recebido um diagnóstico que explica suas alterações profundas de humor, produz certo alívio, reduz o sentimento de culpa, pois acena com a possibilidade de tratamento e controle de seu transtorno e isso pode e deve ser utilizado na escuta terapêutica destes.

Como nas falas acima, "me senti triste" ou "fiquei triste" foram as expressões utilizadas pela maioria dos participantes, para descrever 0 sentimento suscitado pela lembrança de sofrimentos passados ou do relato de perdas antigas. Podemos entender isso como um reviver de mágoas e traumas sofridos no passado, pois todos os entrevistados acima colocaram no passado a responsabilidade frente a seus sintomas depressivos atuais.

Importante frisar aqui que os sintomas descritos acima pelos participantes de nosso estudo podem ocorrer pelo fato dos mesmos terem a percepção mais intensa acerca do sofrimento psíquico ao serem entrevistados do que estes realmente são, fato este relacionado a estados depressivos instalados e corroborados por autores como Silva, Wendt, Argimon ${ }^{17}$, Oliveira, Mazzaia, Marcolan ${ }^{18}$ e Ely, Nunes, Carvalho ${ }^{19}$. Esta sensação de piora dos sintomas depressivos também foi verificado em estudo realizado por Silva et $a^{20}$. Reforçamos que para estes participantes foram realizados orientações e encaminhamentos ao CAPS II do município onde o estudo foi realizado para avaliação.

Notamos nas falas acima mecanismos de racionalização e de negação dos que sofriam com a sintomatologia depressiva. No sentido da racionalização, Blaya et al. ${ }^{21}$ colocam que este é um mecanismo de defesa imaturo da psique humana que trabalha a fim de aceitar a pressão do superego, de disfarçar verdadeiros motivos, de tornar o inaceitável mais aceitável e, enquanto obstáculo ao crescimento, pois a racionalização impede a pessoa de aceitar e de trabalhar com as forças motivadoras genuínas, que causam intensa dor psíquica.

Tavares $^{22}$ afirma ainda que negar a depressão é simbolicamente negar o fracasso do participante na participação da cultura do narcisismo e do espetáculo, pois vivemos em uma cultura em que a imagem tem mais valor do que o ser, na qual o participante passa a viver de um modo particularmente narcisista, pois o eu passa a ser o objeto de investimento libidinal e o outro passa a ser usado como recurso de prazer imediato. Neste sentido, o participante vive em função da imagem, das máscaras que o representam.

Purgato et al..$^{23}$ e Blaya et al. ${ }^{21}$, em seus estudos com participantes depressivos graves, evidenciaram aumento do uso das defesas imaturas, a exemplo da negação e racionalização pelos participantes e que tal uso piorou o prognóstico do transtorno depressivo em longo prazo.

Destacamos que a percepção formada por nossas experiências frente à doença pode ser influenciada por crenças sociais do senso comum, como as menções de alguns dos nossos participantes a relacionar o transtorno depressivo à falta de fé24. Nos estados depressivos, a autopercepção e a capacidade de reconhecer que se sofre de um transtorno mental é tingida e distorcida pelos sentimentos de desamparo, abandono, solidão, vitimização, culpa, perda de autoestima e incapacidade de perceber aspectos positivos compensatórios, devido à redução da sensibilidade ao prazer ${ }^{23}$.

Orbach et al. ${ }^{25}$ apontam ainda que a diminuição da percepção da depressão leva ao agravamento da sintomatologia instalada, aumenta a repulsão pela vida e a atração pela morte, o que pode vir a engatilhar a intenção para cometer o suicídio. Fernandes, Marcolan ${ }^{26}$ colocam que a não percepção dos sintomas depressivos provoca retardo na busca por tratamento e, por consequência, apresenta agravamento do quadro.

Cabe também frisar que, embora estes participantes não se perceberam deprimidos, as avaliações pelas escalas psicométricas apresentaram escores positivos para a sintomatologia depressiva e os mesmos foram encaminhados ao CAPS II do município onde o estudo foi realizado.

No que tange à percepção dos participantes para a relação entre sintomatologia depressiva e comportamento suicida, houve relatos consensuais sobre a mesma. Estudos evidenciam que a dificuldade de percepção adequada frente às causas da depressão e a falta de recursos internos emocionais para lidar com situações estressantes e difíceis são características de indivíduos depressivos e piora conforme a intensidade do quadro, a gerar alteração da autocrítica e piora da sensação de sofrimento psíquico $2,3,9$. Quanto à morte ser verbalizada como solução frente ao intenso sofrimento psíquico desencadeado pelos sintomas vivenciados, devese destacar que tal perspectiva surge nos quadros moderados e graves da depressão ${ }^{6}$, em conexão com nossos resultados, pois o grupo com ausência de sintomas prévios apresentou percentuais maiores para intensidade moderada e grave de sintomatologia depressiva, via escalas psicométricas de avaliação. A literatura menciona ainda, entre os sentimentos característicos da depressão grave, o sentimento de impotência perante à vida e de que não há saída para a agonia psíquica de que se está sofrendo, como um gatilho para o comportamento suicida ${ }^{2,6,9}$. 
Quanto à relação entre desesperança e comportamento suicida, esta é compreendida como um importante gatilho, pois a desesperança configura-se por pensamentos auto derrotistas e a visão pessimista e negativa diante do futuro, e está interligada à depressão ${ }^{27}$, o efeito devastador que a ausência de significado ou propósito de vida causa com seu impacto corrosivo sobre o estado emocional, cognitivo e comportamental ${ }^{28}$, como também observamos nos participantes de nosso estudo. Por outro lado, é bem conhecida a associação entre transtornos de ansiedade e depressão $2,6,9$, uma vez que estudos têm apontado que em média $70 \%$ dos participantes com depressão sofrem concomitantemente de ansiedade e cerca de $80 \%$ dos que têm ansiedade apresentam quadro associado à depressão ${ }^{29,30}$, uma vez que se forma um circuito de retroalimentação entre ansiedade e depressão, a potencializar sintomas e servir de gatilho à desesperança e desejo de morrer.

Outra associação que se fez presente nos relatos, e se encontra na literatura, foi entre sintomatologia depressiva e desemprego, excesso de trabalho e ambiente laboral psiquicamente desgastante, devido excesso de cobranças eo comportamento suicida ${ }^{31,32}$. Ressalta-se que a saúde mental é prejudicada pelas atuais contradições entre modernização e expansão da precarização social e do trabalho, devido à maximização da competitividade que gera intensas cobranças, propiciadora de adoecimentos relacionados ao trabalho, pela violência laboral a que os trabalhadores são submetidos, assim como o próprio desemprego ou subemprego, com aumento da ideação suicida ${ }^{32}$.

Outra associação importante foi a culpa retroalimentada pelo quadro depressivo e desencadeadora de comportamento suicida. Karwowski33 coloca que a ideação de culpa é sintomatologia frequente em quadros depressivos e que quando não tratada adequadamente se torna importante fator para a depressão e o suicídio, uma vez que traz consigo padrões negativos de pensamento.

Faz-se desta forma a importância que os profissionais da área da saúde, em específico o enfermeiro pelas horas de cuidado que detêm junto ao indivíduo depressivo e com comportamento suicida, sejam capacitados para identificar precocemente os sintomas da depressão e ser oferecido tratamento adequado e eficaz ${ }^{33}$, assim como estratégias eficazes de intervenções preventivas a indivíduos com comportamento suicida nas Redes de Atenção à Saúde em todo território nacional ${ }^{2,3,9,34}$.

\section{CONCLUSÃO}

Conclui-se que quanto à autopercepção acerca do sofrimento psíquico, nos chamou a atenção para a ausência de percepção de estar deprimido, a apontar para o agravamento da sintomatologia instalada e aumento para o risco de comportamento suicida e morte.

Deve-se atentar para a ineficácia dos serviços de saúde frente a deteç̧ão precoce dos indivíduos deprimidos, a fim de evitar agravamento do quadro, com cronificação e surgimento da ideação suicida.

Para os que conseguiram ter tal percepção, esta esteve atrelada a situações vivenciadas ao longo da vida, como perdas afetivas, ausência de apoio familiar, perda da autoestima, tristeza, solidão. Surgiu ainda como fator agravante para o comportamento suicida a desesperança, a presença de comorbidade psiquiátrica, como os transtornos de ansiedade e condições de trabalho estressantes e desemprego.

Por fim, entende-se como limitações desta pesquisa a realidade retratada somente na amostra do município de Poços de Caldas/MG, de modo que não possibilita a generalização dos dados para os municípios do entorno da referida cidade. Há de se considerar ainda que estes dados são pioneiros e retratam o panorama da sintomatologia depressiva e sua relação com o comportamento suicida em população adulta de Poços de Caldas/MG, o que permitirá aos gestores municipais a realização de planejamento para o enfrentamento das questões abordadas.

\section{REFERÊNCIAS}

1. Nóbrega IRAP, Leal MCC, Marques APO, Vieira JCM. Fatores associados à depressão em idosos institucionalizados: revisão integrativa. Saúde debate [Internet]. 2015 June [cited 2020 Dec 30]; 39(105): 536-550.

2. World Health Organization. Suicide in the world: Global Health Estimates: Brussels. 2019. https://apps.who.int/iris/bitstream/ handle/10665/326948/WHO-MSD-MER-19.3-eng.pdf?ua=1

3. Marcolan J, Silva D. O comportamento suicida na realidade brasileira: aspectos epidemiológicos e da política de prevenção. Revista M. Estudos sobre a morte, os mortos e o morrer [Internet]. 2019 Sep 1; [Citado em 2020 Dec 30]; 4(7): 31-44.

4. Gondim AS, Coelho Filho JM, Cavalcanti AA, Roriz Filho JS, Nogueira CB, Peixoto Junior AA et al . Prevalence of functional cognitive impairment and associated factors in Brazilian community-dwelling older adults. Dement. neuropsychol. [Internet]. 2017 Mar [cited 2020 Dec 30]; 11(1): 32-39.

5. World Health Organization. Mental Health ATLAS 2017. 2018. World Health Organization. Brussels; https://apps.who.int/iris/bitstream/hand le/10665/272735/9789241514019-eng.pdf?ua=1 
6. American Psychatric Association - APA. Diagnostic an statistical manual of mental disorder, Fifth Editon (DSM - V). Washington: DC. 2015.

7. Bardin L. Análise de conteúdo. São Paulo: Edições 70; 2011. https://docero.com.br/doc/n5v0sv

8. Melo AK, Siebra AJ, Moreira V. Depressão em Adolescentes: Revisão da Literatura e o Lugar da Pesquisa Fenomenológica. Psicologia: Ciência e Profissão. 2017; 37(1), 18-34.

9. World Health Organization. Preventing Suicide - A Global Imperative. Brussels. 2014. https://www.who.int/mental_ health/suicide-prevention/world_report_2014/en/

10. Peluso ETP, Blay SL. Percepção da depressão pela população da cidade de São Paulo. Rev. Saúde Pública [Internet]. 2008 Feb [cited 2020 Dec 30]; 42(1): 41-48.

11. Viana MC, Andrade LH. Lifetime Prevalence, age and gender distribution and age-of-onset of psychiatric disorders in the São Paulo Metropolitan Area, Brazil: results from the São Paulo Megacity Mental Health Survey. Rev. Bras. Psiquiatr. [Internet]. 2012 Oct [cited 2020 Dec 30]; 34(3): 249-260.

12. Lin J, Peruchi MM, Souza LH, Furlanetto LM, Langdon EJ. Percepção e expressão de sintomas depressivos em três grupos culturais catarinenses: açorianos, italianos e alemães. J. bras. psiquiatr. [Internet]. 2008 [cited $2020 \mathrm{Dec} 30$ ]; 57(1): 2-8.

13. Kennedy B, Fang F, Valdimarsdóttir U, Udumyan R, Montgomery S, Fall K. Stress resilience and cancer risk: a nationwide cohort study. J Epidemiol Community Health. 2017;71(10):947-953.

14. Oliveira RM, Siqueira Júnior AC, Furegato ARF. Cuidados de enfermagem ao participante psiquiátrico e ao participante de outras especialidades: percepção da enfermagem. Revista Reme. 2019; 23: e-1198.

15. Gonzáles G, Inocente G, Soares M. Intervenção em grupo para mulheres com endometriose. Psic., Saúde \& Doenças [Internet]. 2019 Ago [citado 2020 Dez 31];20(2): 512-524.

16. Moll MF et al. Nursing professionals and psychiatric admission in general hospital: perceptions and professional training. Cogitare Enferm. 2017; (22): 2:e49933. doi: 10.5380/ce.v22i2

17. Silva MA, Wendt GW, Argimon IIL. Inventário de depressão de beck II: análises pela teoria do traço latente. Aval. psicol. [Internet]. 2018 [citado 2020 Dic 30]; 17(3): 339-350.

18. Oliveira FP, Mazzaia MC, Marcolan JF. Sintomas de depressão e fatores intervenientes entre enfermeiros de serviço hospitalar de emergência. Acta paul. enferm. [Internet]. 2015 June [cited 2020 Dec 30]; 28(3): 209-215.

19. Ely P, Nunes MFO, Carvalho LF. Avaliação psicológica da depressão: levantamento de testes expressivos e autorrelato no Brasil. Aval. psicol. [Internet]. $2014 \mathrm{Dez}$ [citado 2020 Dez 30]; 13(3): 419-426.

20. Silva PO et al. Prevalência de sintomas depressivos e seus fatores associados em idosos atendidos por um centro de referência. Rev. bras. geriatr. gerontol. [Internet]. 2019 [cited 2020 Dec 30]; 22(5): e190088.

21. Blaya C, Kipper L, Heldt E, Isolan L, Ceitlin LH, Bond M et al . Versão em português do Defense Style Questionaire
(DSQ-40) para avaliação dos mecanismos de defesa: um estudo preliminar. Rev. Bras. Psiquiatr. [Internet]. 2004 Dec [cited 2020 Dec 30]; 26(4): 255-258.

22. Tavares C. Estratégias de atuação para a prevenção do suicídio num Serviço de Urgência Geral. Dissertação de Mestrado, Escola Superior de Saúde, Brasil. 2014. https://comum.rcaap.pt/bitstream/10400.26/6274/1/ Tese\%20abril\%202014.pdf

23. Purgato $M$, Gastaldon C, Papola D, van Ommeren M, Barbui C, Tol WA. Psychological therapies for the treatment of mental disorders in low- and middle-income countries affected by humanitarian crises. Cochrane Database Syst Rev. 2018; 7(7): CD011849.

24. Botelho ASC, Pereira MG. Qualidade de vida, otimismo, enfrentamento, morbidade psicológica e estresse familiar em pacientes com câncer colorrectal em quimioterapia. Estudos de Psicologia (Natal). 2015; 20(1), 50-60.

25. Orbach I, Milstein I, Har-Even D, Apter A, Tiano S, Elizur A. A Multi-Attitude Suicide Tendency Scale for adolescents. Psychological Assessment: A Journal of Consulting and Clinical Psychology. 1881; 3(3), 398-404.

26. Fernandes DM, Marcolan JF. Trabalho e sintomatologia depressiva em enfermeiros da Estratégia de Saúde da Família. SMAD Rev Eletr Saúde Mental Álcool Drog [Internet]. 9 de fevereiro de 2017 [citado 30 de dezembro de 2020];13(1):37-44.

27. Marback RF, Pelisoli C. Terapia cognitivo-comportamental no manejo da desesperança e pensamentos suicidas. Revista Brasileira de Terapias Cognitivas. 2014; 10(2), 122-129.

28. Tasca R, Massuda A, Carvalho WM, Buchweitz C, Harzheim E. Recomendações para o fortalecimento da atenção primária à saúde no Brasil. Rev Panam Salud Publica. 2020; 44:e4.

28. Shao R, He $P$, Ling $B$, et al. Prevalence of depression and anxiety and correlations between depression, anxiety, family functioning, social support and coping styles among Chinese medical students. BMC Psychol. 2020; 8(1): 38.

29. Bernard R, Sabariego C, Cieza A. Difficulties Encountered by People With Depression and Anxiety on the Web: Qualitative Study and Web-Based Expert Survey. J Med Internet Res. 2019; 21(10):e12514.

30, Lu YK, Qiao YM, Liang X, et al. Reciprocal relationship between psychosocial work stress and quality of life: the role of gender and education from the longitudinal study of the Survey of Health, Ageing and Retirement in Europe [published correction appears in BMJ Open. 2019 Aug 18;9(8):e027051corr1].

31. Nery BLS, Cruz KCT, Faustino AM, Santos CTB. Vulnerabities, depression and religiosity in elderly hospitized in emergency unit. Rev. Gaúcha Enferm. [Internet]. 2018 [cited 2020 Dec 30]; 39: e2017-0184.

32. Karwowski SL. Por um entendimento do que se chama psicopatologia fenomenológica. Revista da Abordagem Gestáltica. 2015; 21(1), 62-73.

33. Marcolan JF. For a public policy of surveillance of suicidal behavior. Revista Brasileira de Enfermagem. 2018; 71(Supl. 5), 2343-2347. 


\section{Agradecimentos}

Coordenação de Aperfeiçoamento de Pessoal de Nível Superior (CAPES).

\section{Autor Correspondente:}

Giovanna Vallim Jorgetto

gjorgetto1@gmail.com

Editor:

Prof. Dr. Marcelo Riberto

Recebido: 02/01/2021

Aprovado: 25/06/2021 